\title{
Design of 12-phase, 2-stage Harmonic Rejection Mixer for TV Tuners
}

\author{
Dongju LEE, Hocheol JEONG, Minjae LEE \\ School of Information and Communications, Gwangju Institute of Science and Technology, Gwangju, Korea \\ minjae@gist.ac.kr \\ Manuscript received January 21, 2016
}

\begin{abstract}
A two-stage 12-phase harmonic rejection mixer (HRM) for TV tuners is proposed in order to reject the local oscillator (LO) harmonics up to the ninth order. The proposed 12-phase weighing scheme can eliminate the third and ninth harmonic rejection (HR) sensitivity to the amplitude error caused by irrational numbers such as $\sqrt{3}$. To verify this $H R$, the 2-stage HR circuit is designed with baseband $g_{m}$ scaling in order to save power and improve the HR ratios without calibration. The proposed HRM achieves the third to ninth worst HR ratios, more than $55 \mathrm{~dB}$, according to Monte Carlo simulations. It consumes $6.5 \mathrm{~mA}$ under a $2.5 \mathrm{~V}$ supply voltage.
\end{abstract}

\section{Keywords}

Harmonic rejection mixer, multiphase, TV tuner, direct conversion, active mixer

\section{Introduction}

For wideband analog/digital TV tuners covering VHF and UHF bands, harmonic rejection (HR) is a challenging issue because the radio frequency (RF) signals and unwanted signals around the local oscillator (LO) harmonics are translated into the same baseband.

The required $\mathrm{HR}$ ratios for $\mathrm{TV}$ are $60-70 \mathrm{~dB}$ for $\mathrm{LO}$ harmonics [1]. In order to suppress the unwanted harmonic mixing, various HR techniques have been introduced [1-4]; however, HR ratios are degraded due to the amplitude and phase errors that result from device mismatch [2]. In order to reduce the sensitivity of the HR to mismatch, 2-stage HR techniques [5], [6] have been presented. The 2-stage harmonic mixing has two merits: amplitude error reduction and small integer numbers for root number generation.

1) In 2-stage harmonic mixing [5], the total amplitude error from the HR stages is a multiplication of the errors in the first and second HR stages: $\left(\varepsilon_{1 \mathrm{st}} \cdot \varepsilon_{2 \mathrm{nd}}\right) / 4$, where $\varepsilon_{1 \mathrm{st}}$ and $\varepsilon_{2 \text { nd }}$ are the relative errors in the first/ second HR stages, respectively. Therefore, this 2-

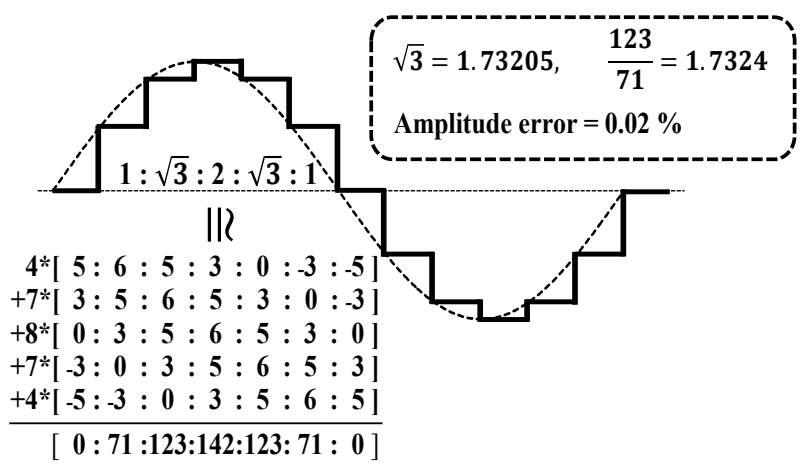

Fig. 1. The effective LO waveform of the proposed HRM.

stage harmonic mixing can reduce the HR sensitivity to stage amplitude errors.

2) In order to have a weighting ratio of $\{0: 29: 41: 29\}$ for an 8-phase LO with 2-stage, weighting numbers in each stage range only between 2 to $7 ;\{2: 3: 2\} /\{5: 7: 5\}$. Thus, the unit finger width of the transistors can be easily increased to reduce device mismatch.

However, these 2-stage HR techniques use 8-phase LOs in which, particularly for VHF (54-216 MHz) band reception, the odd harmonics higher than the fifth order also down-convert unwanted signals to baseband because input RF frequencies span up to $16 \mathrm{X}$ range (from 54 to $860 \mathrm{MHz}$ ). To ease the requirements of the RF filter and to extend HR to the ninth harmonic, the number of LO phases must be increased to 12. Conventional 12-phase LO, however, has been reported with 1-stage implementation [7], of which HR ratios are vulnerable to amplitude mismatch or device mismatch.

In this paper, a 12-phase harmonic rejection mixer (HRM) with a 2-stage is proposed to desensitize HR ratios to device mismatch so that the third, fifth, seventh, and ninth HR is realized for TV tuner bands (54-860 MHz). To the extent of our knowledge, a 2-stage HRM with 12-phase LOs has not been reported before. This paper is organized as follows. Section 2 describes the 12-phase HR principle, numerical calculations of HR ratios, and circuit implementations of the HRM. The Monte Carlo simulation results are presented and discussed in Sec. 3. Finally, the paper is concluded in Sec. 4. 


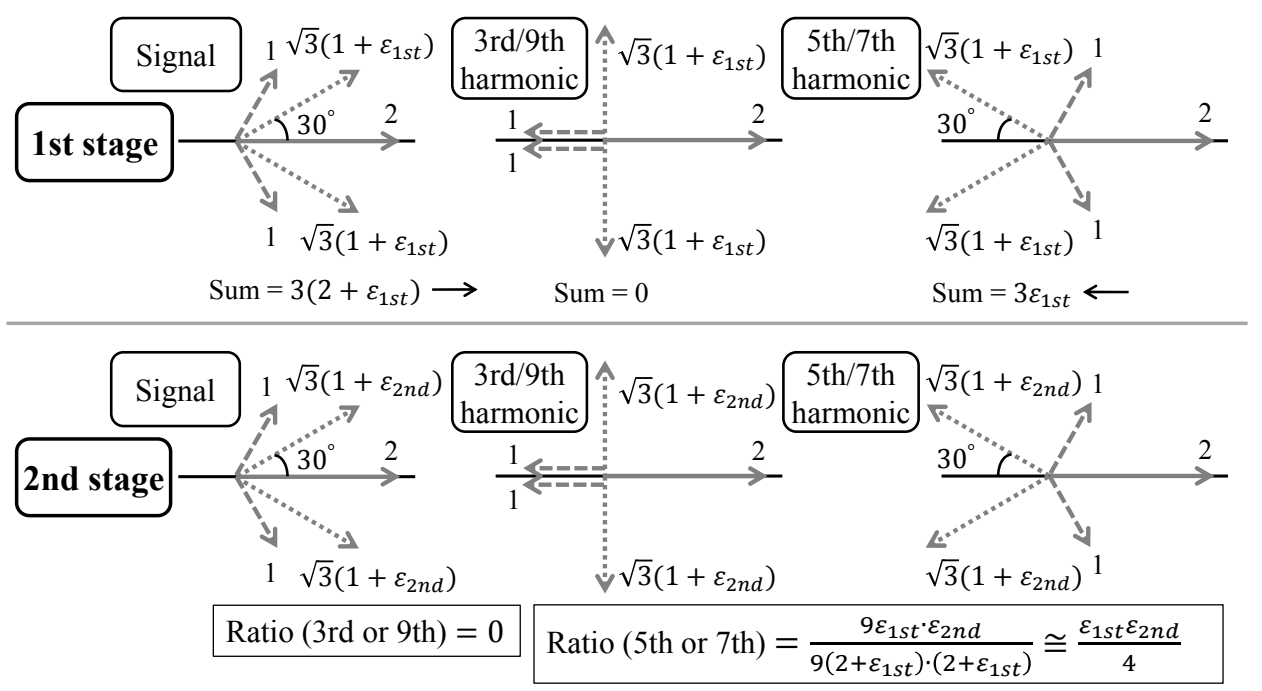

Fig. 2. The vector diagrams of 12-phase 2-stage harmonic mixing.

\section{Harmonic Mixing}

\subsection{Design Consideration}

When the number of non-overlapping LO phases is $N$, the LO harmonics greater than or equal to the $(N-1)^{\text {th }}$ order are not suppressed, which results in poor HR ratios, e.g. only $20 \log (n) \mathrm{dB}$ for the $n$th harmonic. Thus, a 12-phase $\mathrm{LO}$ is required in order to enable the sine wave to reject the third to ninth LO harmonics, whereas the 8-phase LO [5], [6] can only reject third/fifth LO harmonics. A 12-phase HRM [7] based on LO-gating techniques [8], [9] was realized through 1-stage harmonic mixing with a weighting ratio of $\{6.2: 10.8\}$ to approximate $\sqrt{3}$. This $\{31: 54\}$ ratio is close to $\sqrt{3}$ with a $0.57 \%$ amplitude error. However, it is not easy to implement these high integer numbers with good matching and low power consumption in 1-stage harmonic mixing particularly when transconductance $\left(g_{\mathrm{m}}\right)$ scaling [2] is used. The similar issue exists in 8-phase HRMs as well and has been overcome by 2 -stage harmonic mixing [5].

To design the 12-phase 2-stage HR circuit, the static amplitude error due to the irrational number $(\sqrt{3})$ limits the achievable HR ratio and should be considered. When the total weighting ratio in order to generate $\{1: \sqrt{3}: 2: \sqrt{3}: 1\}$ is selected, it is not easy to find corresponding integer weighting ratios for each stage in the 2stage HR circuit. First, possible small integer weighing ratios to approximate $\{1: \sqrt{3}: 2: \sqrt{3}: 1\}$ are investigated. Then, weighting ratios for each stage can be extended as follows: $\{2: 3: 4: 3: 2\},\{3: 5: 6: 5: 3\},\{4: 7: 8: 7: 4\}, \cdots$, and so on. Two selections among those can make the desired total weighting ratio for the 2-stage HR. In this paper, the $\{3: 5: 6: 5: 3\} /\{4: 7: 8: 7: 4\}$ ratios were selected to generate $\{0: 71: 123: 142: 123: 71\}$, as depicted in Fig. 1. The zero factor in this ratio is realized from the cancellation of differential circuits. As the weighting factors increase, the ratio between the first and second factors approaches $\sqrt{3}$, but higher factors require more areas and power for the $g_{m}$ scaling technique.

Figure 2 depicts the effects of the relative errors, $\varepsilon_{1 \mathrm{st}}$ and $\varepsilon_{2 n d}$ on the approximations for $\sqrt{3}$. The relative errors of third/ninth harmonics are perfectly cancelled despite the non-zero $\varepsilon_{1 \text { st }}$ and $\varepsilon_{2 \text { nd }}$ because the total vector summation for the third/ninth harmonics becomes zero, which results from the error vectors having equal amplitude and opposite sign. The cancellation of these relative errors helps to improve the third HR ratio (HR3) and HR9. In the case of the fifth and seventh harmonics, the total relative error can be reduced to $\varepsilon_{1 \mathrm{st}} \cdot \varepsilon_{2 \text { nd }} / 4$. The achievable HR5 or HR7 is the sum of the HR ratios in the each HR stage. The $\varepsilon_{1 \mathrm{st}}$ in a $\{3: 5\}$ ratio for the approximation of $\sqrt{3}$ is $3.775 \%$ and the $\varepsilon_{2 \text { nd }}$ in a $\{4: 7\}$ ratio is $1.03 \%$. Then, the HR ratios in the first and second HR stages are $34.5 \mathrm{~dB}$ and $45.76 \mathrm{~dB}$, respectively. The total HR ratio is $80.26 \mathrm{~dB}$, when no phase error is assumed.

\subsection{HR Sensitivity Analysis to Amplitude and Phase Errors}

When both amplitude and phase errors are considered, the HR ratios are estimated through equations based on [5]. The HR3 of a single-balanced 12-phase HRM is derived as

$$
\frac{\sin ^{2}(3 \pi / 12)}{162 \sin ^{2}(\pi / 12)}\left[\sigma_{A}^{2}+27(1+\varepsilon)^{2} \sigma_{\theta}^{2}\right]
$$

where $\sigma_{\mathrm{A}}$ is the standard deviation (SD) of amplitude error in percentage, $\sigma_{\theta}$ is the $\mathrm{SD}$ of phase error in radian, and $\varepsilon$ is the relative error due to the approximation of $\sqrt{3}$ in percentage. From (1), it is noted that $\sigma_{\mathrm{A}}$ is not multiplied by $\varepsilon$ since the two $\sqrt{3}$ vectors in the third/ninth harmonics are in the opposite direction as described in Fig. 2. Thus, HR3 is less sensitive to $\sigma_{\mathrm{A}}$ and dominated by $\sigma_{\theta}$ in our scheme. 


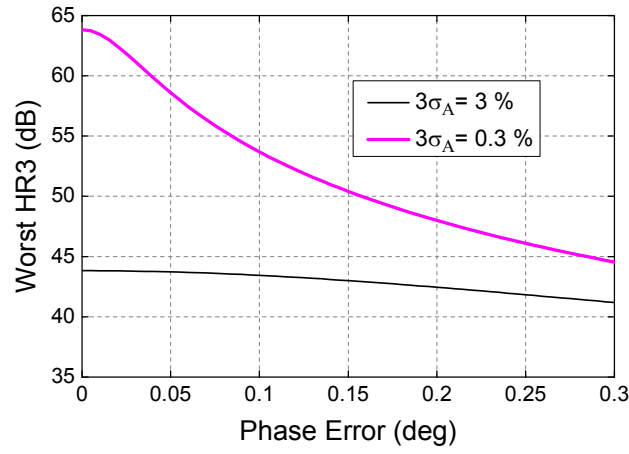

(a)

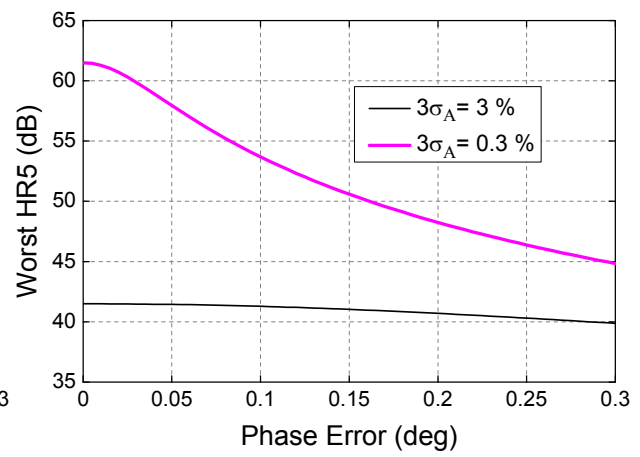

(b)

Fig. 3. Estimated (a) HR 3 and (b) HR5 versus phase error with $3 \sigma_{\mathrm{A}}=3 \%$ and $0.3 \%$ for a 12 -phase HRM.

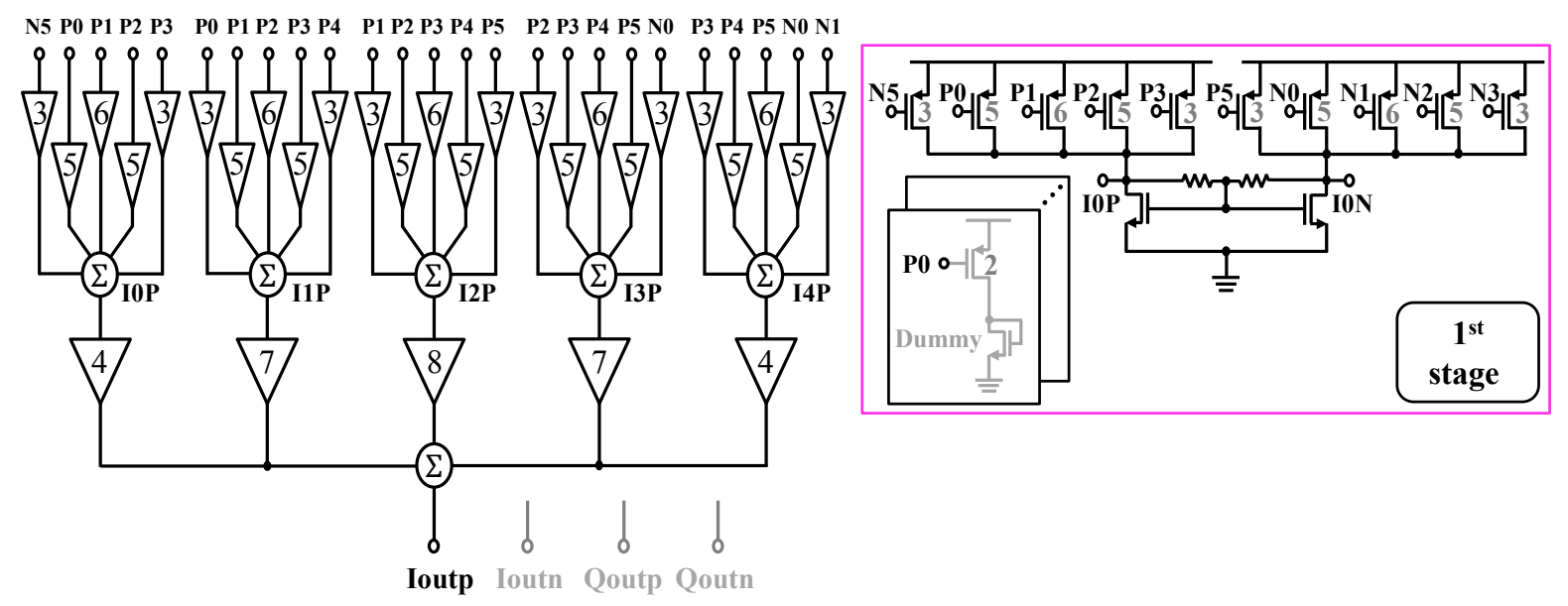

Fig. 4. The 12-phase HR circuit.

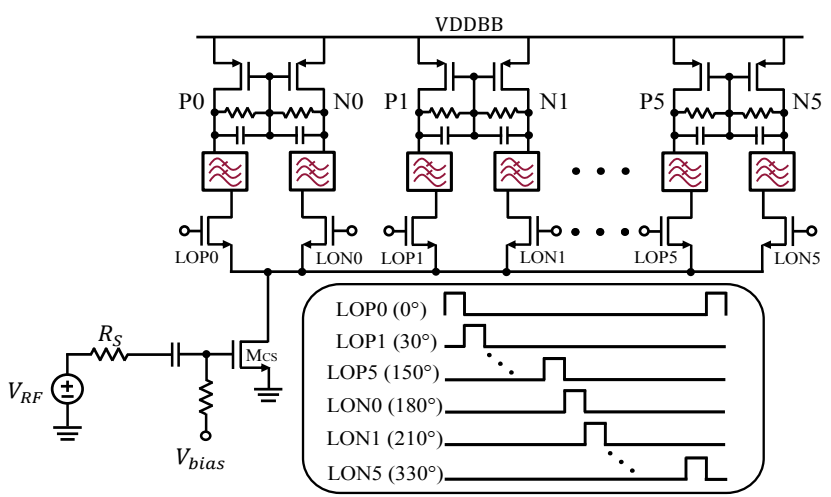

Fig. 5. The RF section of the HRM.

Similarly, the HR5 of a 12-phase HRM is written as

$\frac{\sin ^{2}(5 \pi / 12)}{1800 \sin ^{2}(\pi / 12)}\left[\left(1+9(1+\varepsilon)^{2}\right) \sigma_{\mathrm{A}}^{2}+75\left(1+(1+\varepsilon)^{2}\right) \sigma_{\theta}^{2}\right]$

Assuming $\varepsilon=1 \%, 3 \sigma_{\mathrm{A}}$ worst-case $\mathrm{HR}$ ratios versus phase error are plotted in Fig. 3. When phase error is zero, the $3 \sigma_{\mathrm{A}}(0.3 \%)$ worst HR3 and HR5 are 63.8 and $61.5 \mathrm{~dB}$. To accomplish $\mathrm{HR}$ ratios over than $50 \mathrm{~dB}, 3 \sigma_{\mathrm{A}}$ amplitude error is selected to $0.3 \%$ and $\sigma_{\theta}$ should be less than $0.15^{\circ}$.

\subsection{Implementation of HRM}

Two methods are conventionally used in baseband HR circuits: the resistor weighting with OTAs and $\mathrm{g}_{\mathrm{m}}$ scaling. Although resistor matching is more accurate than $\mathrm{g}_{\mathrm{m}}$ matching, the number of OTAs and power consumption increases significantly. Unlike OTAs, which require a constant bias current, the common-source (CS) amplifier with $g_{m}$ scaling only passes the signal current when the LO switch is on and the branch is active, which results in less current consumption. Thus, the $\mathrm{g}_{\mathrm{m}}$-based HR circuit was implemented in this work. The block diagram of the 2stage HR circuit for the I and Q channels is presented in Fig. 4. In the first HR stage, PMOS amplifiers have a $g_{m}$ ratio of $\{3: 5: 6: 5: 3\}$ in order to approximate $\{1: \sqrt{3}: 2: \sqrt{3}: 1\}$.

This $g_{m}$ ratio is implemented by scaling the total width of MOSFETs. As an instance, the device size for the factor 3 is $W / L=37.5 \mu \mathrm{m} / 1.2 \mu \mathrm{m}$. But the problem is that each PMOS amplifier in the first stage has different $\mathrm{Cgs}$ due to their different total width, which increases path mismatch and degrades HR performances. To eliminate this mismatch, dummy cells with diode-connected NMOS 


\begin{tabular}{|c|c|c|c|c|c|c|c|c|c|c|c|c|c|}
\hline & P0 & P1 & P2 & P3 & P4 & P5 & N0 & N1 & N2 & N3 & N4 & N5 & \\
\hline I0P-I0N & 5 & 6 & 5 & 3 & 0 & -3 & -5 & -6 & -5 & -3 & 0 & 3 & $\cdot 4$ \\
\hline I1P-I1N & 3 & 5 & 6 & 5 & 3 & 0 & -3 & -5 & -6 & -5 & -3 & 0 & $\cdot 7$ \\
\hline I2P-I2N & 0 & 3 & 5 & 6 & 5 & 3 & 0 & -3 & -5 & -6 & -5 & -3 & $\cdot 8$ \\
\hline I3P-I3N & -3 & 0 & 3 & 5 & 6 & 5 & 3 & 0 & -3 & -5 & -6 & -5 & $\cdot 7$ \\
\hline I4P-I4N & -5 & -3 & 0 & 3 & 5 & 6 & 5 & 3 & 0 & -3 & -5 & -6 & $\cdot 4$ \\
\hline Total & 0 & 71 & 123 & 142 & 123 & 71 & 0 & -71 & -123 & -142 & -123 & -71 & \\
\hline
\end{tabular}

Tab. 1. Weighting factors for 12-phase harmonic mixing.

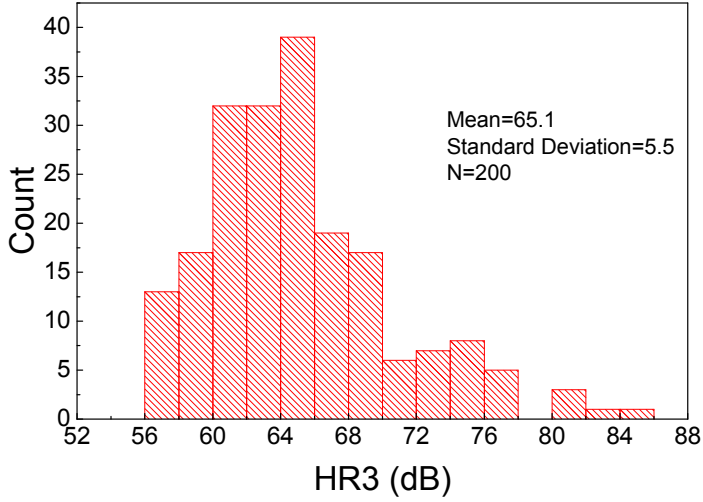

(a)

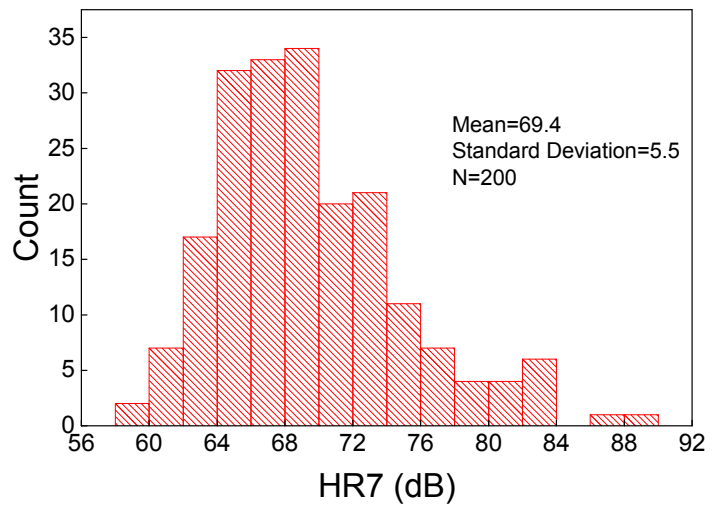

(c)

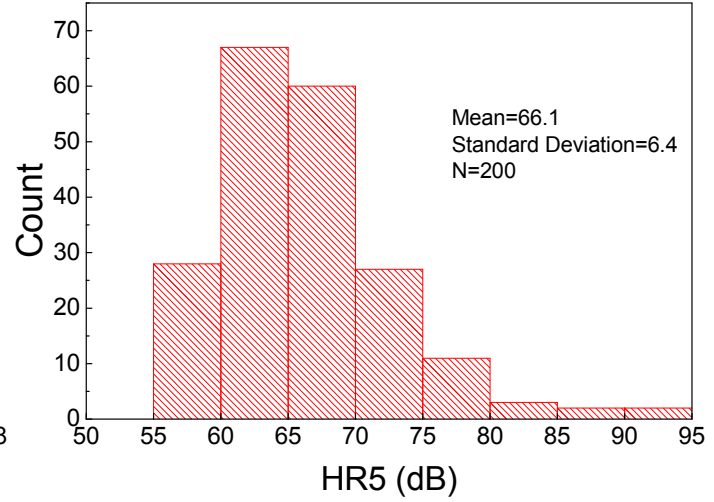

(b)

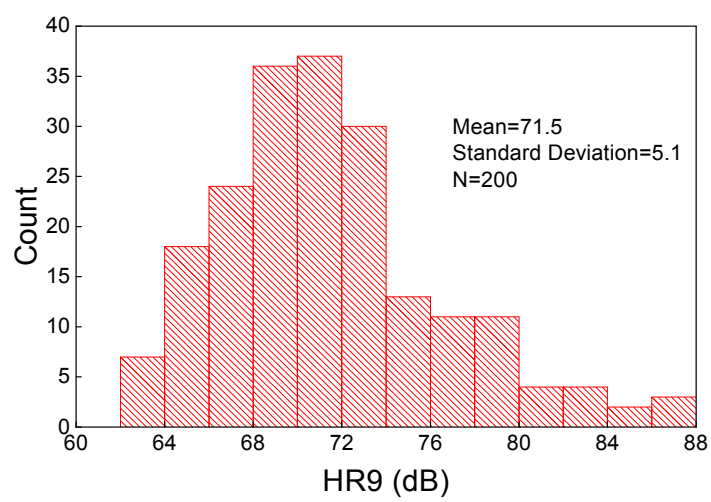

(d)

Fig. 6. Monte Carlo simulation results of (a) HR3, (b) HR5, (c) HR7, and (d) HR9 for the proposed 12-phase 2-stage HRM with the weighting ratio of $\{3: 5: 6: 5: 3\} /\{4: 7: 8: 7: 4\}$ at the $111 \mathrm{MHz}$ effective LO.

loads in Fig. 4 are added to match loading capacitances in the paths. The combined drain currents at the first stage outputs are converted to differential voltages via load resistors, which drive the NMOS CS amplifiers in the second $\mathrm{HR}$ stage that has a $\mathrm{g}_{\mathrm{m}}$ ratio of $\{4: 7: 8: 7: 4\}$ for five $30^{\circ}$ phase-shifted signals. These weighting factors are summarized in Tab. 1.

The RF section of the HRM is described in Fig. 5. The CS amplifier $\left(\mathrm{M}_{\mathrm{CS}}\right)$ generates a bias current of $1 \mathrm{~mA}$ in order to drive the 12-phase active mixer. The 12-phase LO switches are driven using non-overlapping 8.33\% dutycycle LO signals. The lowpass filters (LPF) are connected to the mixer in order to suppress the LO feedthrough and out-of-band interferers. The mixer, LPFs, and thick PMOS loads operate under a $2.5 \mathrm{~V}$ supply for better linearity.

\section{Simulation Results}

HR performances depend on both amplitude and phase errors of the effective LO waveform. In the $\mathrm{g}_{\mathrm{m}}$-based HR circuit, the LO amplitude is realized by $\mathrm{g}_{\mathrm{m}}$ scaling that is sensitive to device mismatch. Thus, the Monte Carlo HR simulations are used to estimate their sensitivity to device mismatch and different process corners as well. In order to find the HR ratios, an RF signal $(111 \cdot N+4 \mathrm{MHz}, N=1$, $3,5,7$, and 9) was applied and down-converted to $4 \mathrm{MHz}$ via the $N^{\text {th }}$ order LO harmonics with the $111 \mathrm{MHz}$ effective LO. Then, the power differences between the fundamental and harmonic tones were compared. Figure 6 illustrates that the worst-case third, fifth, seventh, and ninth HR ratios obtained by Monte Carlo simulation are 56, 55, 58, and $62 \mathrm{~dB}$, respectively. 


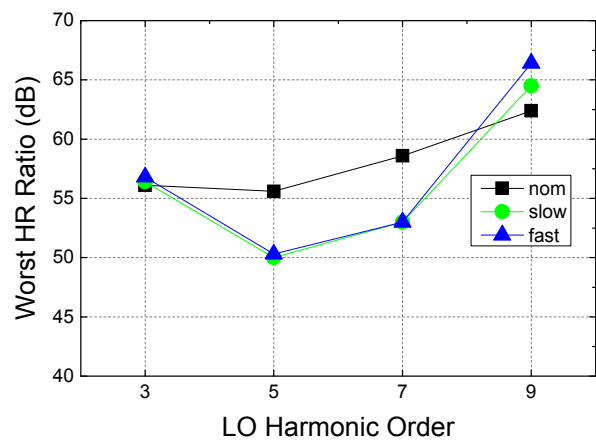

(a)

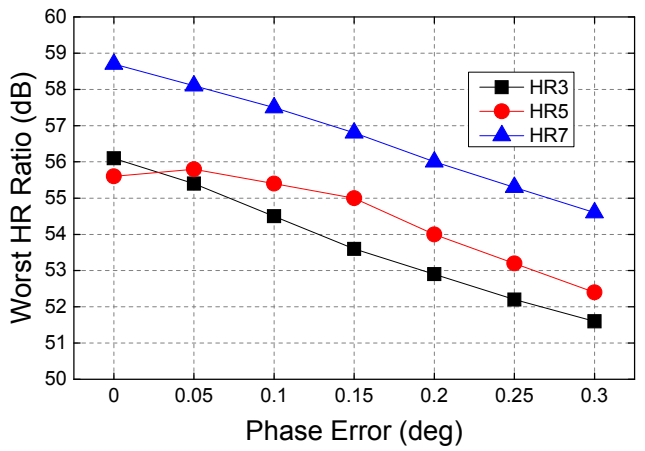

(b)

Fig. 7. Worst-case HR ratios from Monte Carlo simulation results (200 runs) with (a) process corners and (b) phase error at the $111 \mathrm{MHz}$ effective LO.

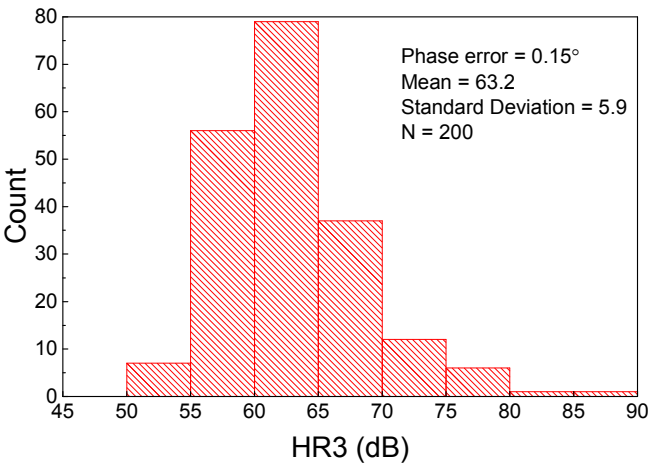

(a)

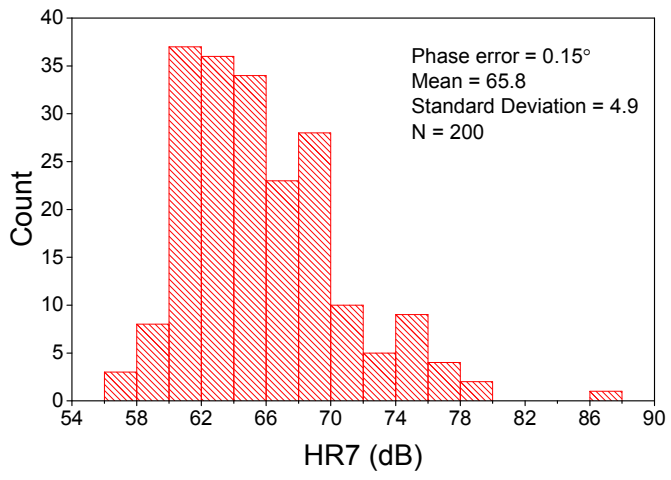

(c)

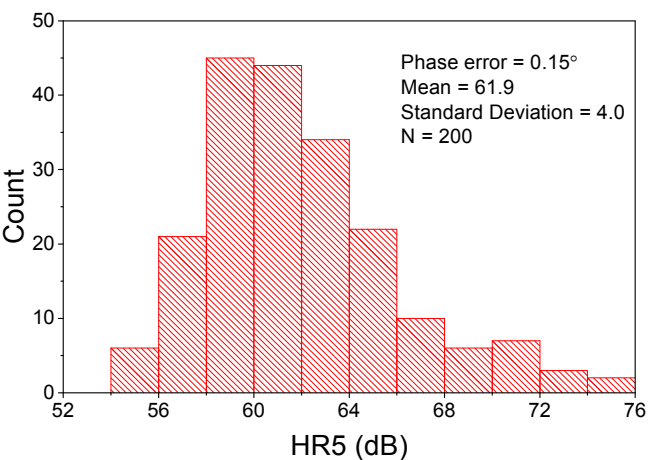

(b)

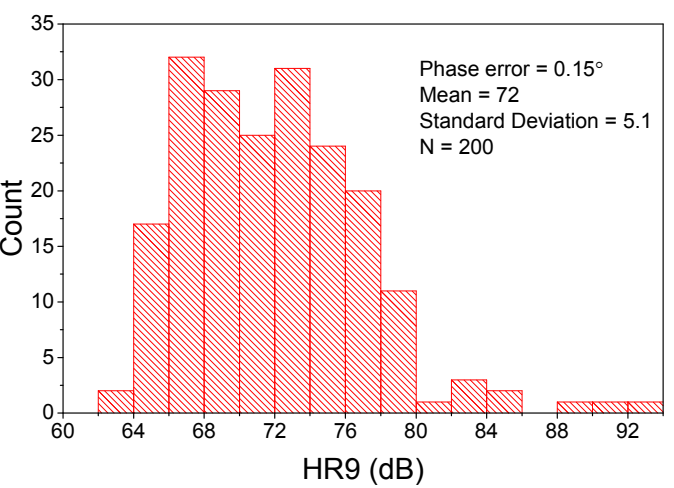

(d)

Fig. 8. Monte Carlo simulation results of (a) HR3, (b) HR5, (c) HR7, and (d) HR9 including $0.15^{\circ}$ phase error at the $111 \mathrm{MHz}$ effective LO.

This $\mathrm{g}_{\mathrm{m}}$-based 2 -stage HR circuit has $\sim 0.1 \%$ drain current mismatch due to $\mathrm{g}_{\mathrm{m}}$ scaling according to Monte Carlo simulation. Derived from (1) and (2), the estimated $3 \sigma$ worst-case HR3 and HR5 are supposed to be 63.8 and $61.5 \mathrm{~dB}$, which are $\sim 7 \mathrm{~dB}$ higher than the Monte Carlo simulation results shown in Fig. 6. These discrepancies result from unaccounted mismatch sources other than $g_{m}$ scaling mismatch, such as mixer switches and loads.

Figure 7 shows the effects of process corners and phase error in HR ratios. Figure 7(a) demonstrates that the worst-case $\mathrm{HR}$ ratios maintain $>50 \mathrm{~dB}$ in nom $\left(27{ }^{\circ} \mathrm{C}\right)$, slow $\left(120^{\circ} \mathrm{C}\right)$, and fast $\left(-40^{\circ} \mathrm{C}\right)$ corners. In Fig. $7(\mathrm{~b})$, the worst-case HR ratios are still better than $50 \mathrm{~dB}$ even with $0.3^{\circ}$ phase error. The phase error is generated by phase shifts of the LO signal. The Monte Carlo simulation results assuming phase error of $0.15^{\circ}$ are plotted in Fig. 8, where the worst-case HR3 of 53.6 and HR 5 of $55 \mathrm{~dB}$ are achieved.

The wideband HR ratios of the proposed HRM are shown in Fig. 9. The RF signal at $115 \mathrm{MHz}$ is down-converted to $4 \mathrm{MHz}$ by the $111 \mathrm{MHz}$ effective LO and the third RF interference at $335 \mathrm{MHz}$ is down-converted to $2 \mathrm{MHz}$ by the third LO harmonic. The third to ninth RF interferences at low frequency bands $(54-95 \mathrm{MHz})$ are also down-converted to in-band by LO harmonics, thus HR3 to 


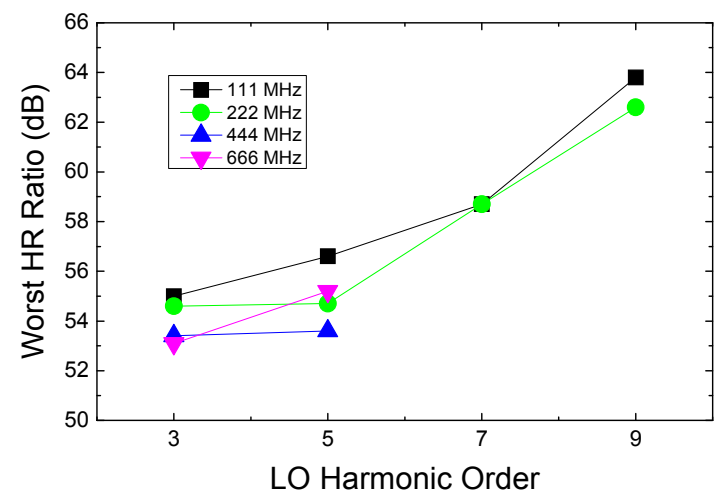

Fig. 9. Worst-case HR3-9 with third to ninth RF interferences.

\begin{tabular}{|c|c|c|c|}
\hline & 12-phase HRM & 8-phase HRM & This Work \\
\hline Architecture & $\begin{array}{c}\text { 12-phase } \\
\text { 1-stage }\end{array}$ & $\begin{array}{c}\text { 8-phase } \\
\text { 2-stage }\end{array}$ & $\begin{array}{c}12 \text {-phase } \\
\text { 2-stage }\end{array}$ \\
\hline $\begin{array}{c}\text { Weighting } \\
\text { ratios }\end{array}$ & $\begin{array}{c}\{0: 15: 26: 30 \\
: 26: 15\}\end{array}$ & $\begin{array}{c}\{2: 3: 2\} / \\
\{5: 7: 5\}\end{array}$ & $\begin{array}{c}\{3: 5: 6: 5: 3\} / \\
\{4: 7: 8: 7: 4\}\end{array}$ \\
\hline DSB NF (dB) & 10.3 & 10.5 & 11 \\
\hline $\begin{array}{c}\text { Voltage gain } \\
\text { (dB) }\end{array}$ & 22.7 & 41.5 & 40.3 \\
\hline IIP3 (dBm) & -1.5 & +0.6 & -0.5 \\
\hline $\begin{array}{c}\text { HR3, 5, 7,9 } \\
(\mathbf{d B})\end{array}$ & $52 / 52 / 56 / 61$ & $56 / 59 / 17 / 21$ & $56 / 55 / 58 / 62$ \\
\hline Power (mW) & 4.7 & 11.75 & 16.25 \\
\hline
\end{tabular}

Tab. 2. Performance summary and comparison.

HR9 are reported. The effective LO frequency is varied from 111 to $666 \mathrm{MHz}$. The worst-case HR3 is maintained greater than $53 \mathrm{~dB}$.

The HRM performances are summarized and compared in Tab. 2. Worst-case HR ratios from Monte Carlo simulations (200 runs) are reported in Tab. 2. The device size for the biggest factor 30 in the 1-stage HRM is $W / L=$ $75 \mu \mathrm{m} / 1.2 \mu \mathrm{m}$, which is the same size as the factor 6 in the proposed HRM. The 2-stage harmonic mixing improves HR3 by $4 \mathrm{~dB}$ compared to 1 -stage harmonic mixing. Compared to the 8-phase HRM, the proposed 12-phase HRM achieves almost $40 \mathrm{~dB}$ improvement on HR7 and HR9, while maintaining comparable voltage gain and IIP3. Under a $2.5 \mathrm{~V}$ supply, the 8-phase and proposed 12-phase HRMs consumed 11.75 and $16.25 \mathrm{~mW}$, respectively. Although the 12-phase HRM requires more power consumption compared to the 8-phase HRM, it can reject odd harmonics up to the ninth order.

\section{Conclusion}

A 12-phase HRM using the 2-stage $\mathrm{g}_{\mathrm{m}}$-based HR circuit is presented for the TV tuner bands in order to suppress unwanted odd LO harmonics. Unlike conventional 8-phase HR schemes, the proposed 12-phase 2-stage HR scheme extends the maximum order of HR from the fifth order to the ninth order harmonic with small weighting factors, and rejects the third to the ninth LO harmonics between 55 to $62 \mathrm{~dB}$ without calibration. Although our scheme was demonstrated on the $\mathrm{g}_{\mathrm{m}}$ scaling scheme, it can be also applied to other resistor weighting schemes with transimpedance amplifiers (TIAs).

\section{Acknowledgments}

This research was supported by Basic Science Research Program through the National Research Foundation of Korea (NRF) funded by the Ministry of Science, ICT and Future Planning (2013R1A1A1009259). The CAD tools were supported by IDEC.

\section{References}

[1] CHEN, C., WU, J., HUANG, C., et al. A CMOS switched load harmonic rejection mixer for DTV tuner application. IEEE Transactions on Circuits and Systems I: Regular Papers, 2013, vol. 60, no. 2, p. 428-436. DOI: 10.1109/ TCSI.2012.2215695

[2] WELDON, J., NARAYANASWAMI, R., RUDELL, J., et al. A $1.75-\mathrm{GHz}$ highly integrated narrow-band CMOS transmitter with harmonic-rejection mixers. IEEE Journal of Solid-State Circuits, 2001, vol. 36, no. 12, p. 2003-2015. DOI: 10.1109/4. 972151

[3] LERSTAVEESIN, S., GUPTA, M., KANG, D., et al. A 48-860 MHz CMOS low-IF direct-conversion DTV tuner. IEEE Journal of Solid-State Circuits, 2008, vol. 43, no. 9, p. 2013-2024. DOI: 10.1109/JSSC.2008.2001900

[4] ANDREWS, C., DIAMENTE, L., YANG, D., et al. A wideband receiver with resonant multi-phase $\mathrm{LO}$ and current reuse harmonic rejection baseband. IEEE Journal of Solid-State Circuits, 2013, vol. 48, no. 5, p. 1188-1198. DOI: 10.1109/JSSC. 2013.2254535

[5] RU, Z., MOSELEY, N., KLUMPERINK, E., et al. Digitally enhanced software-defined radio receiver robust to out-of-band interference. IEEE Journal of Solid-State Circuits, 2009, vol. 44, no. 12, p. 3359-3375. DOI: 10.1109/JSSC.2009.2032272

[6] LIN, F., MAK, P.-I., MARTINS, R. An RF-to-BB-current-reuse wideband receiver with parallel $\mathrm{N}$-path active/passive mixers and a single-MOS pole-zero LPF. IEEE Journal of Solid-State Circuits, 2014, vol. 49, no. 11, p. 2547-2559. DOI: 10.1109/JSSC.2014.2354647

[7] RAFI, A. A., VISWANATHAN, T.R. Harmonic rejection mixing techniques using clock-gating. IEEE Journal of Solid-State Circuits, 2013, vol. 48, no. 8, p. 1862-1874. DOI: 10.1109/JSSC.2013.2259032

[8] PUllela, R., SOWLATI, T., ROZENBlit, D. Low flickernoise quadrature mixer topology. In IEEE International Solid-State Circuits Conference, ISSCC Digest of Technical Papers. San Francisco (USA), 2006, p.1870-1879. DOI: 10.1109/ ISSCC.2006.1696244

[9] LEE, D., LEE, M. Low flicker noise, odd-phase master LO active mixer using a low switching frequency scheme. IEEE Journal of Solid-State Circuits, 2015, vol. 50, no. 10, p. 2281-2293. DOI: 10.1109/JSSC.2015.2449556

\section{About the Authors ...}

Dongju LEE was born in 1983. He received his M.S. degree from Gwangju Institute of Science and Technology 
(GIST), Gwangju, in 2008. He is currently working towards the Ph.D. degree at GIST. His research interests include analog and RF IC designs for multiband and multimode wireless receivers.

Hocheol JEONG was born in 1988. He received the B.S. degree in Electronic and Radio Wave Engineering from Kyunghee University (KHU), Suwon, Korea in 2014, the M.S. degree from GIST, Gwangju, Korea in 2016. He is currently working toward the Ph.D. degree at GIST. His research areas are analog and RF IC design for wireless applications.

Minjae LEE was born in 1976. He received his B.Sc. and M.S. degrees both in Electrical Engineering from Seoul National University, Seoul, Korea in 1998 and 2000 respectively. He received the Ph.D. degree in Electrical Engi- neering from the University of California, Los Angeles, in 2008. In 2000, he was a consultant with GCT semiconductor, Inc., and Silicon Image Inc., designing analog circuits for wireless communication and digital signal processing blocks for Gigabit Ethernet. He joined Silicon Image Inc., Sunnyvale, CA in 2001, developing Serial ATA products. In August 2008, he joined Agilent Technologies in Santa Clara, CA, where he was involved with the development of next generation high-speed ADCs and DACs. Since 2012, he has been with the School of Information and Communications, Gwangju Institute of Science and Technology, Gwangju, Korea, where he is now an Assistant Professor. He was the recipient of the 2007 Best Student Paper Award at the VLSI Circuits Symposium in Kyoto, Japan. He received the 2015 Distinguished Lecture Award in Gwangju Institute of Science and Technology. 